\title{
Avaliação do risco de sangramento na profilaxia do tromboembolismo venoso
}

\author{
Bleeding risk assessment for venous thromboembolism prophylaxis \\ Maria Chiara Chindamo ${ }^{1,2}$ (D), Marcos Arêas Marques ${ }^{3}$ (1)
}

\begin{abstract}
Resumo
O tromboembolismo venoso (TEV) é uma das principais causas preveníveis de morbimortalidade em pacientes hospitalizados, sendo a embolia pulmonar (EP) fatal possivelmente a sua primeira manifestação. Diretrizes nacionais e internacionais recomendam o uso de modelos de avaliação de risco para a prescrição de profilaxia do TEV em pacientes hospitalizados. Apesar das evidências e diretrizes de apoio, o uso da tromboprofilaxia permanece abaixo do ideal, o que pode resultar da baixa conscientização dos benefícios da profilaxia, mas também pode refletir o medo de complicações hemorrágicas, justificando a subutilização da tromboprofilaxia em todo o mundo. A avaliação do risco de sangramento é, portanto, necessária para a adequação de profilaxia e deve ser realizada de forma concomitante à avaliação do risco de trombose. O objetivo desta revisão é salientar a importância da avaliação conjunta do risco de TEV e do risco de sangramento em pacientes hospitalizados.
\end{abstract}

Palavras-chave: trombose venosa; embolia pulmonar; profilaxia; hemorragia; segurança do paciente; avaliação de risco.

\begin{abstract}
Venous thromboembolism (VTE) is one of the main preventable causes of morbidity and mortality in hospitalized patients and fatal pulmonary embolism (PE) may be its first manifestation. Several national and international guidelines recommend using risk assessment models for prescription of VTE prophylaxis in hospitalized patients. Despite evidence and guidelines supporting VTE prevention, use of VTE prophylaxis in hospitalized patients remains suboptimal, which may be because of low awareness of the benefits of VTE prophylaxis, but might also reflect fear of bleeding complications in these patients, since this constitutes one of the main reasons for underutilization of thromboprophylaxis worldwide. Bleeding risk assessment is therefore necessary for adequate prophylaxis prescription and should be carried out concurrently with assessment of the risk of thrombosis. The purpose of this review is to highlight the importance of jointly assessing risk of VTE and risk of bleeding in hospitalized patients.
\end{abstract}

Keywords: venous thrombosis; pulmonary embolism; prophylaxis; hemorrhage; patient safety; risk assessment.

Como citar: Chindamo MC, Marques MA. Avaliação do risco de sangramento na profilaxia do tromboembolismo venoso. J Vasc Bras. 2021;20:e20200109. https://doi.org/10.1590/1677-5449.200109

\footnotetext{
'Universidade Federal do Rio de Janeiro - UFRJ, Rio de Janeiro, RJ, Brasil.

${ }^{2}$ Hospital Barra D'Or - Rede D'Or São Luiz, Rio de Janeiro, RJ, Brasil.

${ }^{3}$ Universidade do Estado do Rio de Janeiro - UERJ, RJ, Brasil.

Fonte de financiamento: Nenhuma.

Conflito de interesse: Os autores são palestrantes da Sanofi.

Submetido em: Junho 17, 2020. Aceito em: Outubro 13, 2020.
}

O estudo foi realizado no Hospital Barra D'Or - Rede D'Or São Luiz, Rio de Janeiro, RJ, Brasil.

Copyrightđ 2021 Os autores. Este é um artigo publicado em acesso aberto (Open Access) sob a licença Creative Commons Attribution, que permite uso, distribuição e reprodução em qualquer meio, sem restrições desde que o trabalho original seja corretamente citado. 


\section{INTRODUÇÃO}

O tromboembolismo venoso (TEV) é uma das principais causas preveníveis de morbimortalidade em pacientes hospitalizados ${ }^{1,2}$. A embolia pulmonar (EP) fatal é frequentemente a primeira manifestação de TEV e pode representar até $10 \%$ de todas as mortes hospitalares ${ }^{2,3}$. Os pacientes hospitalizados apresentam risco de TEV devido à presença de fatores adquiridos ou hereditários, como obesidade, câncer, TEV prévio, trombofilias, trauma, cirurgia, infarto agudo do miocárdio, acidente vascular cerebral, idade avançada, insuficiência cardíaca congestiva, infecção aguda, imobilidade e admissão em unidades de terapia intensiva, entre outros ${ }^{4-6}$.

Diretrizes nacionais e internacionais recomendam o uso de modelos de avaliação de risco [risk assessment model (MAR)] para a profilaxia farmacológica ou mecânica em pacientes clínicos ${ }^{7-14}$, cirúrgicos ${ }^{8,15,16}$ ou obstétricos ${ }^{10}$, visando à melhor estratégia de prevenção. Contudo, o risco de TEV não pode ser avaliado isoladamente. $\mathrm{O}$ risco de sangramento, induzido ou potencializado pelos anticoagulantes, também deve ser avaliado simultaneamente quando se considera a adequação da tromboprofilaxia ${ }^{6,7,9}$.

Embora diversos estudos reportem baixas taxas de sangramento relacionadas à farmacoprofilaxia ${ }^{11,17,18}, \mathrm{o}$ medo de eventos hemorrágicos é uma das principais justificativas da sua subutilização em todo o mundo ${ }^{6}$. A identificação de condições com potencial risco de sangramento e a implementação de MAR são, portanto, fundamentais para o uso adequado da tromboprofilaxia ${ }^{19}$.

O objetivo desta revisão é salientar a importância da avaliação conjunta do risco de TEV e do risco de sangramento em pacientes hospitalizados.

\section{AVALIAÇÃO DE RISCO TROMBÓTICO VERSUS RISCO DE SANGRAMENTO}

Inúmeros MARs de TEV estão disponíveis, tanto para pacientes clínicos quanto cirúrgicos, e direcionam as principais recomendações de tromboprofilaxia com base na estratificação de risco ${ }^{7-16}$. O modelo mais adequado de avaliação ainda não está definido ${ }^{19}$. $\mathrm{Na}$ estratificação de risco de TEV, devemos utilizar um modelo validado para a população em questão aplicado de forma sistemática nas principais etapas de assistência: admissão hospitalar, transição entre setores e alta hospitalar. Essa última avaliação é particularmente importante nos pacientes que mantêm fatores de risco para TEV na alta, como, por exemplo, a imobilidade prolongada ${ }^{2}$. A escolha da melhor estratégia de tromboprofilaxia deve considerar, simultaneamente, o risco de TEV e o risco potencial de sangramento ${ }^{20,21}$.
São consideradas contraindicações absolutas ao uso de anticoagulantes o sangramento ativo, grave ou potencialmente fatal, não reversível com intervenção médica ou cirúrgica, incluindo qualquer sangramento ativo em local crítico (intracraniano, pericárdico, retroperitoneal, intraocular, intra-articular e intraespinhal), hipertensão arterial maligna não controlada, coagulopatia grave não compensada, disfunção plaquetária ou distúrbios primários de hemostasia graves, trombocitopenia persistente $\left(<20.000 / \mathrm{mm}^{3}\right)$ e procedimentos invasivos de alto risco em local crítico, como punção lombar e raquianestesia em pacientes cujo procedimento cirúrgico é planejado no período de 6 a 12 horas seguintes ${ }^{20}$. Outros fatores associados ao risco aumentado de sangramento são a trombocitopenia induzida pela heparina (TIH), o uso concomitante de antiagregantes plaquetários e/ou anti-inflamatórios não hormonais e a disfunção renal, principalmente quando utilizados anticoagulantes de eliminação renal [heparina de baixo peso molecular (HBPM) e fondaparinux $]^{15}$. Em pacientes que apresentam clearance de creatinina $<30 \mathrm{~mL} / \mathrm{min}$, recomenda-se a redução da dose de HBPM, monitoramento da ação anticoagulante ou substituição por heparina não fracionada $(\mathrm{HNF})^{4}$. A revisão periódica de ambos os riscos, principalmente nas mudanças de quadro clínico, ajuda a orientar a melhor estratégia para a profilaxia ${ }^{21}$ (Figura 1).

\section{Modelos de avaliação de risco de TEV}

Entre os principais MARs de TEV em pacientes clínicos, estão a Diretriz Brasileira de Prevenção de TEV em pacientes clínicos hospitalizados ${ }^{9} \mathrm{e}$ os escores de Padua ${ }^{11}$, Genebra ${ }^{13}$ e IMPROVE (International Medical Prevention Registry on Venous Thromboembolism $)^{12}$. Para a avaliação de pacientes cirúrgicos, são recomendados os escores de Caprini ${ }^{15}$ e Rogers ${ }^{16}$, que definem o risco de TEV com base nas características dos pacientes e no perfil de cada cirurgia. Mulheres hospitalizadas durante a gravidez, no puerpério ou no período de até seis semanas após aborto espontâneo ou interrupção da gravidez, devem ser avaliadas quanto à necessidade de farmacoprofilaxia ${ }^{21}$. O MAR mais utilizado nesse perfil de pacientes foi desenvolvido pelo Royal College of Obstetricians and Gynecologists (RCOG) ${ }^{10,21}$.

Apesar da recomendação de avaliação concomitante do risco de TEV versus risco de sangramento durante o cuidado do paciente hospitalizado, são escassos os MARs de sangramento no contexto da profilaxia de TEV $^{19}$. Poucos são os modelos que associam essas duas características ${ }^{9,12,21}$ (Tabela 1).

Esse cenário é bastante distinto daquele encontrado na avaliação de risco de sangramento aplicada à anticoagulação plena na prevenção dos fenômenos tromboembólicos da fibrilação atrial ou no tratamento 

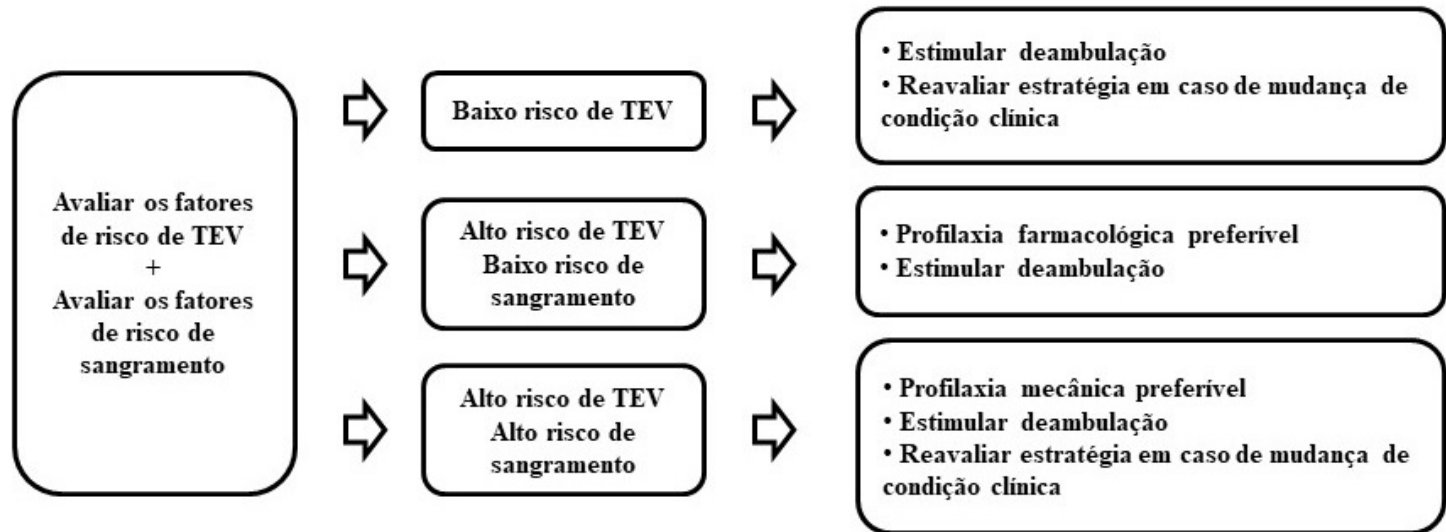

Figura 1. Recomendações de profilaxia de tromboembolismo venoso (TEV) segundo estratificação do risco de TEV versus risco de sangramento. Adaptado de: National Institute for Health and Care Excellence - NICE. NG8921.

Tabela 1. Lista de modelos de avaliação de risco (MAR) de tromboembolismo venoso (TEV) e de sangramento de acordo com a população estudada ${ }^{9-16,21}$.

\begin{tabular}{|c|c|c|c|}
\hline MAR & Tipo de paciente & Risco de sangramento & Recomendação de profilaxia \\
\hline Caprini, 2005 & Cirúrgico & - & $x$ \\
\hline Geneva, 2006 & Clínico & - & $x$ \\
\hline Rogers, 2007 & Cirúrgico & - & $x$ \\
\hline Diretriz brasileira, 2007 & Clínico & $x$ & $x$ \\
\hline Padua, 2010 & Clínico & - & $x$ \\
\hline IMPROVE, 2011 & Clínico & $x$ & $x$ \\
\hline UK RCOG, 2015 & Obstétrico & - & $x$ \\
\hline NICE NG89, 2018 & Clínico/cirúrgico & $x$ & $x$ \\
\hline
\end{tabular}

de TEV. Dez MARs de sangramento estão disponíveis com esse objetivo. Seis deles são aplicáveis aos casos dos pacientes que fazem uso de anticoagulantes orais na fibrilação atrial $\left(\mathrm{ABC}^{22}\right.$, $\mathrm{ORBIT}^{23}$, $\mathrm{ATRIA}^{24}$, HAS-BLED $^{25}$, HEMORR ${ }_{2}$ HAGES $^{26}$, Shireman ${ }^{27}$ ), três no tratamento anticoagulante de TEV (VTE-BLED ${ }^{28}$, Ruiz-Gimenez ${ }^{29}$, Kuijer $^{30}$ ) e um misto $\left(\mathrm{OBRI}^{31}\right)$. Esses escores evidenciam situações de aumento do risco de sangramento associado à anticoagulação plena e auxiliam na aplicação de estratégias que ajudam a minimizar o risco de hemorragia por meio da intervenção em fatores de risco modificáveis ${ }^{32}$.

\section{RISCO DE SANGRAMENTO NA FARMACOPROFILAXIA}

\section{Avaliação de risco de sangramento em pacientes clínicos}

\section{IMPROVE Bleeding Risk Score}

$\mathrm{O}$ principal MAR de sangramento associado à farmacoprofilaxia em pacientes clínicos hospitalizados é o IMPROVE Bleeding Risk Score ${ }^{6,7}$. Decousus et al. ${ }^{6}$ utilizaram uma análise multivariada para identificar e pontuar fatores na admissão hospitalar associados ao risco de sangramento em pacientes clínicos agudamente doentes. Com base nos dados do IMPROVE ${ }^{12}$, um estudo observacional multicêntrico desenvolvido para avaliar os padrões de profilaxia de TEV em mais de 15.000 pacientes clínicos, os autores verificaram a incidência de sangramento e identificaram os fatores, na admissão, associados ao risco de sangramento ${ }^{6}$. O sangramento maior foi definido como sangramento fatal e/ou sintomático em uma área ou órgão crítico e/ ou sangramento que resultou em queda de hemoglobina $\geq 2 \mathrm{~g} / \mathrm{dL}$ ou levou à transfusão de dois ou mais concentrados de hemácias ${ }^{6}$. O sangramento não maior, mas clinicamente relevante, foi caracterizado como hemorragia gastrointestinal, hematúria macroscópica de duração $>24$ h, epistaxe substancial com necessidade de intervenção, epistaxe recorrente e/ou de duração de pelo menos cinco minutos, hematomas extensos ( $>5 \mathrm{~cm}$ de diâmetro), sangramento intra-articular, menorragia ou metrorragia ou ainda outro sangramento importante que necessitasse de intervenção médica ${ }^{6,33}$. 
A incidência cumulativa de sangramentos hospitalares descritos como maiores e não maiores até 14 dias após a internação foi de 3,2\% (1,2\% de sangramento maior e $2,0 \%$ de sangramento não maior clinicamente relevante) $)^{6}$.

Os fatores de risco, na admissão, independentemente associados ao risco de sangramento foram ${ }^{6}$ : úlcera gastroduodenal ativa, sangramento nos três meses anteriores à admissão e contagem de plaquetas $<50.000 / \mathrm{mm}^{3}$. Outros fatores de risco de sangramento incluíram idade avançada, insuficiência hepática e/ou renal, permanência em unidade de terapia intensiva, presença de cateter venoso central, doença reumática, câncer e sexo masculino, fatores que também estão relacionados ao aumento do risco de $\mathrm{TEV}^{6}$. Cada um dos fatores acima, com ponderação apropriada, foi inserido neste MAR (Tabela 2). Os autores também elaboraram um recurso on-line no qual o risco de sangramento pode ser avaliado ${ }^{34}$.

Mais da metade dos episódios de sangramento maior ocorreu em 10\% dos pacientes hospitalizados que tiveram escore de risco de sangramento $\geq 7^{6}$. Desse modo, os autores consideraram como alto risco de sangramento a pontuação no IMPROVE Bleeding Risk Score $\geq 7$ e como baixo risco a pontuação $<7$. As taxas de sangramento maior, em comparação às taxas de qualquer sangramento (definido como maior ou não maior clinicamente relevante) nos pacientes com escore $<7$ foram respectivamente $0,4 \%$ e $1,5 \%$. Naqueles com escore $\geq 7$, a taxa de sangramento maior foi de $4,1 \%$ e a de qualquer sangramento, $7,9 \%{ }^{6}$.

Profilaxia mecânica foi mais utilizada em pacientes com escore de sangramento $\geq 7$, comparada ao escore $<7$ ( $16,3 \%$ versus $8,9 \%$, respectivamente). Por outro lado,

Tabela 2. IMPROVE Bleeding Risk Score.

\begin{tabular}{|c|c|}
\hline Fatores de risco & Pontuação \\
\hline Úlcera gastroduodenal ativa & 4,5 \\
\hline Hemorragia três meses antes da internação & 4 \\
\hline Plaquetas $<50.000 \mathrm{~mm}^{3}$ & 4 \\
\hline Idade $\geq 85$ anos versus. $<40$ anos & 3,5 \\
\hline Insuficiência hepática (RNI* > 1,5) & 2,5 \\
\hline $\begin{array}{l}\text { Insuficiência renal severa (TFG } \text { TF* }^{*}<30 \text { versus. } \geq \\
60 \mathrm{~mL} / \mathrm{min} \text { ) }\end{array}$ & 2,5 \\
\hline Internação em unidade de terapia intensiva & 2,5 \\
\hline Cateter venoso central & 2 \\
\hline Doença reumatológica & 2 \\
\hline Câncer ativo & 2 \\
\hline Idade $40-84$ versus $<40$ anos & 1,5 \\
\hline Sexo masculino & 1 \\
\hline $\begin{array}{l}\text { Insuficiência renal moderada (TFG** } 30-59 \text { versus } \\
\geq 60 \mathrm{~mL} / \mathrm{min} \text { ) }\end{array}$ & 1 \\
\hline
\end{tabular}

a profilaxia farmacológica foi utilizada em proporções semelhantes em pacientes com escore de risco de $<7$ e $\geq 7$ (48,9\% versus $49,3 \%$, respectivamente $)^{6}$.

Este MAR ajuda, portanto, a decidir sobre a profilaxia farmacológica ou mecânica em pacientes clínicos de alto risco de $\mathrm{TEV}^{6}$. Pode ser aplicado em associação com o escore IMPROVE para risco de $\mathrm{TEV}$, permitindo a ponderação do risco e do benefício na escolha da melhor estratégia de tromboprofilaxia. Esse escore já foi validado em outras populações de pacientes clínicos 35,36

\section{Avaliação de risco de sangramento em pacientes cirúrgicos}

A taxa de sangramento associada à farmacoprofilaxia em pacientes cirúrgicos varia de acordo com o perfil da cirurgia. Uma metanálise de 52 estudos randomizados sobre farmacoprofilaxia de TEV em pacientes de cirurgia geral relatou que os sangramentos menores são comuns e abrangem hematomas no local de aplicação $(\sim 7 \%)$, hematoma da ferida $(\sim 6 \%)$, sangramento no local do dreno $(\sim 2 \%)$ e hematúria $(\sim 2 \%)^{37}$. As complicações hemorrágicas maiores, no entanto, foram incomuns e incluíram sangramento gastrointestinal $(0,2 \%)$ ou retroperitoneal $(<0,1 \%)^{37}$. A descontinuação da profilaxia ocorreu em $2 \%$ dos pacientes e a reoperação subsequente por sangramento em menos de $1 \%$. Da mesma forma, os pacientes com um ou mais fatores de risco individuais de sangramento foram considerados de alto risco no pós-operatório ${ }^{37}$.

\section{Estimativas do risco inicial de sangramento em cirurgia}

$\mathrm{O}$ risco inicial de sangramento foi pouco estudado em pacientes cirúrgicos não ortopédicos. A estratificação de risco de sangramento maior foi estimada pelo American College of Chest Physicians (ACCP) nos seguintes grupos de pacientes cirúrgicos: geral/abdominal/ pélvica $(\sim 1 \%)$, bariátrica $(<1 \%)$, plástica/reconstrutiva $(0,5$ a $1,8 \%)$, vascular $(0,4$ a $1,8 \%)$, cardíaca $[\sim 5 \%$ (alto risco)], torácica (1\%), neurocirurgia/craniotomia (1 a $1,5 \%)$, coluna vertebral $(<0,5 \%)$ e trauma grave [3,4 a $4,7 \%$ (alto risco)].

Nas cirurgias ortopédicas, as estimativas de risco inicial de sangramento na ausência de profilaxia são heterogêneas devido às características distintas das populações e das técnicas cirúrgicas utilizadas ${ }^{38}$. Estima-se um risco de sangramento maior, variando de 2 a 4\% nas cirurgias ortopédicas com duração acima de 45 minutos e na artroplastia bilateral de joelhos. Procedimentos menores, como artroscopias, cirurgias de ombro, mãos e pés, são considerados de menor risco de sangramento $(<2 \%)^{39}$. As taxas de sangramento maior em pacientes submetidos a 
profilaxia de TEV variaram de $0,1 \%$ a $3,1 \%$ nos estudos de artroplastia de quadril e de $0,2 \%$ a $1,4 \%$ nos estudos de artroplastia de joelho, sugerindo haver pouca interferência dos anticoagulantes no risco de sangramento desses grupos de pacientes ${ }^{40}$.

\section{Risco de sangramento em situações especiais}

\section{Trombocitopenia}

As diretrizes atuais sobre profilaxia de TEV são baseadas em ensaios clínicos randomizados que excluem os indivíduos de risco potencialmente alto de sangramento, limitando, portanto, as recomendações específicas sobre farmacoprofilaxia em pacientes com trombocitopenia e/ou disfunção plaquetária ${ }^{41}$. Essas condições estão presentes em pelo menos 25\% dos indivíduos hospitalizados e são representadas por uma série de patologias, como púrpura trombocitopênica idiopática, púrpura trombocitopênica trombótica, síndrome do anticorpo antifosfolipídeo (SAF), TIH, coagulação intravascular disseminada, plaquetopenia induzida por medicamentos, insuficiência hepática, renal e medular e câncer ${ }^{41}$. Os valores mínimos de plaquetas para a utilização da farmacoprofilaxia também não são uniformes, variando entre 50.000 e $100.000 / \mathrm{mm}^{3}$. ${ }^{6,9,11,15,21}$. O IMPROVE Bleeding Risk Score ${ }^{6}$ considera como referência o limite de plaquetas de $50.000 / \mathrm{mm}^{3}$, enquanto a diretriz $\mathrm{NICE}^{21}$ estabelece o ponto de corte em 75.000/ $\mathrm{mm}^{3}$. O risco de sangramento espontâneo aumenta dramaticamente quando há contagens de plaquetas de $<10.000$ a $20.000 / \mathrm{mm}^{3}$ e varia de acordo com a causa da trombocitopenia ${ }^{41}$.

\section{Hepatopatia crônica}

A trombocitopenia ou disfunção plaquetária associadas a anormalidades da coagulação são comuns em pacientes com cirrose hepática ${ }^{41}$. No entanto, esses pacientes apresentam alta incidência de trombose venosa portal e idiopática, o que significa que a coagulopatia da cirrose não protege contra a trombose ${ }^{41}$. As situações associadas à trombocitopenia leve/ moderada $\left(>50.000 / \mathrm{mm}^{3}\right)$ não devem interferir nas decisões de prevenção de TEV. Na trombocitopenia grave $\left(<50.000 / \mathrm{mm}^{3}\right)$, no entanto, a profilaxia deve ser considerada individualmente ${ }^{41}$. Em uma revisão sistemática sobre tromboprofilaxia e trombocitopenia, Tufano et al. ${ }^{41}$ propõem recomendações especificas para o uso de profilaxia farmacológica (Tabela 3).

\section{Síndrome anticorpo antifosfolipídeo (SAF)}

Em pacientes com SAF e trombocitopenia, a tendência trombótica é geralmente muito superior ao risco de sangramento ${ }^{41}$. A profilaxia de TEV deve ser avaliada nessa população, especialmente
Tabela 3. Estratégia de prevenção do TEV em pacientes com cirrose e/ou trombocitopenia.

\begin{tabular}{|c|c|}
\hline $\begin{array}{c}\text { Risco de sangramento } \\
\text { espontâneo }\end{array}$ & Recomendações \\
\hline $\begin{array}{l}\text { Baixo (plaquetas } \\
<90.000 \mathrm{~mm}^{3} \text { ) }\end{array}$ & Profilaxia farmacológica* \\
\hline $\begin{array}{l}\text { Intermediário (plaquetas ente } \\
50 \text { e } 90.000 \mathrm{~mm}^{3} \text { ) }\end{array}$ & Profilaxia farmacológica* \\
\hline \multirow{2}{*}{ Alto (plaquetas $<50.000 \mathrm{~mm}^{3}$ ) } & $\begin{array}{l}\text { Profilaxia farmacológica em } \\
\text { casos selecionados* }\end{array}$ \\
\hline & $\begin{array}{l}\text { Profilaxia mecânica } \\
\text { preferencial }{ }^{* *}\end{array}$ \\
\hline
\end{tabular}

*A profilaxia do TEV deve ser realizada na presença de um ou mais fatores de risco adicionais para TEV; ${ }^{* *}$ Meias elásticas de compressão graduada, compressão pneumática intermitente e dispositivos e bombas podais. Adaptada de: Tufano et al. ${ }^{41}$.

naqueles considerados de alto risco, como, por exemplo, os pacientes positivos para os três anticorpos antifosfolipídios: anticoagulante lúpico, anticardiolipina e anti 32 glicoproteína I (triplo-positivo) ${ }^{41}$. O Global APS Score (GAPSS) é um MAR que analisa o perfil dos anticorpos antifosfolipídios e fatores de risco cardiovascular e pode ser útil para avaliar o risco de eventos trombóticos em pacientes portadores de lúpus eritematoso sistêmico, porém ainda não foi validado ${ }^{42}$.

Até $30 \%$ dos pacientes portadores de SAF podem apresentar trombocitopenia $\left(<100.000 / \mathrm{mm}^{3}\right)$, porém o sangramento é raro e normalmente está associado à SAF catastrófica, trombocitopenia imune ou naqueles que desenvolvem anticorpos contra protrombina ou outros fatores da coagulação ${ }^{42}$.

\section{Pacientes oncológicos}

O câncer é um fator de risco independente e importante para o desenvolvimento de $\mathrm{TEV}^{43}$. Por outro lado, pacientes com câncer são propensos a sangramentos, associados às complicações dos tumores, frequência aumentada de procedimentos cirúrgicos e trombocitopenia associada à quimioterapia sistêmica, tornando a prevenção do TEV um grande desafio nessa população. A profilaxia do TEV deve ser considerada em pacientes hospitalizados com câncer mesmo na presença de trombocitopenia, principalmente nos que têm múltiplos fatores de risco para $\mathrm{TEV}^{43,44}$. Nos pacientes que apresentam valores de plaquetas $>80.000 / \mathrm{mm}^{3}$, recomenda-se o uso de farmacoprofilaxia em dose padrão ${ }^{43,44}$. Se a contagem de plaquetas estiver abaixo de $80.000 / \mathrm{mm}^{3}$, a conduta deve ser considerada individualmente ${ }^{43,44}$. Recomenda-se monitoramento cuidadoso quanto a efeitos indesejáveis relacionados ao uso do anticoagulante versus risco de $\mathrm{TEV}^{43,44}$. No caso de contraindicação à profilaxia farmacológica, deve-se otimizar o uso da profilaxia mecânica. 
Insuficiência Renal Crônica (IRC)

A IRC é uma doença de caráter paradoxal do ponto de vista da coagulação, pois, ao mesmo tempo em que aumenta o risco de TEV devido à injúria/ disfunção endotelial, hiperreatividade plaquetária inicial, aumento da formação de fibrina e diminuição da atividade do sistema fibrinolítico, aumenta o risco de hemorragia maior com a piora progressiva da função renal e consequente decréscimo da adesão e agregação plaquetária ${ }^{45}$. Embora o IMPROVE Bleeding Risk Score ${ }^{6}$ pontue a IRC de acordo com a sua gravidade (um ponto para IRC moderada e 2,5 pontos para IRC severa), os MARs de TEV para pacientes clínicos (Diretriz Brasileira de Prevenção-de TEV ${ }^{9}$ e os escores de Padua ${ }^{11}$, Genebra $^{13}$ e IMPROVE ${ }^{12}$ ) e pacientes cirúrgicos (escore de Caprini ${ }^{15}$ e Rogers ${ }^{16}$ ) não incluem a IRC como fator de risco de trombose. Esse equilíbrio frágil entre o risco aumentado de TEV e o risco de hemorragia torna a farmacoprofilaxia do TEV um desafio particular especialmente naqueles com IRC avançada (clearance de creatinina 15$29 \mathrm{~mL} / \mathrm{min}$ ) ou falência renal terminal (clearance de creatinina $<15 \mathrm{~mL} / \mathrm{min}$ ), por várias razões, incluindo o fato de não haver um MAR específico para esse grupo de pacientes ${ }^{45}$.

Com relaçao às opções de profilaxia farmacológica, as evidências são insuficientes para concluir que o uso de HNF na dose de 5.000 UI três vezes/dia aumente o risco de eventos hemorrágicos graves e menores em pacientes com clearence de creatinina $<30 \mathrm{~mL} / \mathrm{min}$ em comparação a pacientes sem disfunção renal severa ou que a enoxaparina aumente significativamente o risco de sangramento maior em comparação a HNF nesse perfil de pacientes ${ }^{46}$.

\section{Como proceder no caso de pacientes com aumento do risco de sangramento}

No caso dos pacientes hospitalizados que apresentam alto risco de TEV associado a alto risco de sangramento ou que têm contraindicação do uso de anticoagulantes, os métodos mecânicos de prevenção de TEV, como compressão pneumática intermitente, meias de compressão graduadas e bomba venosa plantar são recomendados ${ }^{6}$. Quando as opções de profilaxia mecânica são utilizadas, a transição para um agente farmacológico deve ser considerada assim que o risco de sangramento se tornar baixo ou for revertido.

\section{CONCLUSÃO}

A adequação do uso de farmacoprofilaxia deve estar alinhada com a minimização do risco de sangramento para que os pacientes classificados como de alto risco de desenvolvimento de TEV obtenham benefício clínico real da tromboprofilaxia.

Diversas diretrizes de prevenção de TEV orientam sobre os principais fatores implicados no risco de sangramento. No entanto, até o momento, o único MAR validado que permite a identificação dos pacientes clínicos na admissão hospitalar sob risco de sangramento é o IMPROVE Bleeding Risk Score ${ }^{6,36}$. Os pacientes com pontuação $<7$ podem receber farmacoprofilaxia com segurança ${ }^{6}$. Por outro lado, pacientes com alto risco de sangramento (pontuação $\geq 7$ ), mas que apresentam simultaneamente alto risco de TEV, devem ter as decisões relativas à profilaxia avaliadas de forma individualizada e dinâmica ao longo da internação, até a alta hospitalar. Nos pacientes submetidos à cirurgia, é necessário considerar o potencial risco de sangramento do procedimento em conjunto com os fatores de risco individuais para definir a melhor estratégia de prevenção de TEV.

\section{REFERÊNCIAS}

1. Geerts WH, Bergqvist D, Pineo GF, et al. Prevention of venous thromboembolism: American College of Chest Physicians EvidenceBased Clinical Practice Guidelines, 8th ed. Chest. 2008;133(6, Supl):381S-453S. http://dx.doi.org/10.1378/chest.08-0656. PMid:18574271.

2. Caprini JA. Identification of patient venous thromboembolism risk across the continuum of care. Clin Appl Thromb Hemost. 2011;17(6):590-9. http://dx.doi.org/10.1177/1076029611404217. PMid:21593024.

3. Sandler DA, Martin JF. Autopsy proven pulmonary embolism in hospital patients: are we detecting enough deep vein thrombosis? J R Soc Med. 1989;82(4):203-5. http://dx.doi. org/10.1177/014107688908200407. PMid:2716016.

4. Geerts WH, Pineo GF, Heit JA, et al. Prevention of venous thromboembolism: the Seventh ACCP Conference on Antithrombotic and Thrombolytic Therapy. Chest. 2004;126(3, Supl):338S-400S. http://dx.doi.org/10.1378/chest.126.3_suppl.338S. PMid:15383478.

5. Cohen AT, Tapson VF, Bergmann JF, et al. Venous thromboembolism risk and prophylaxis in the acute hospital care setting (ENDORSE study): a multinational cross-sectional study. Lancet. 2008;371(9610):387-94. http://dx.doi.org/10.1016/S0140-6736(08)60202-0. PMid:18242412.

6. Decousus H, Tapson VF, Bergmann JF, et al. Factors at admission associated with bleeding risk in medical patients: findings from the IMPROVE investigators. Chest. 2011;139(1):69-79. http:// dx.doi.org/10.1378/chest.09-3081. PMid:20453069.

7. Kahn SR, Lim W, Dunn AS, et al. Prevention of VTE in nonsurgical patients: antithrombotic therapy and prevention of thrombosis, 9th ed: American College of Chest Physicians Evidence-Based Clinical Practice Guidelines. Chest. 2012;141(2, Supl):e195S-226S. http://dx.doi.org/10.1378/chest.11-2296. PMid:22315261.

8. Gould MK, Garcia DA, Wren SM, et al. Prevention of VTE in nonorthopedic surgical patients antithrombotic therapy and prevention of thrombosis, 9th ed: American College of Chest Physicians Evidence-Based Clinical Practice Guidelines. Chest. 2012;141(2, Supl):e227S-77S. http://dx.doi.org/10.1378/chest.11-2297. PMid:22315263. 
9. Rocha AT, Paiva EF, Lichtenstein A, Milani RJr, Cavalheiro CF, Maffe $\mathrm{FH}$. Risk-assessment algorithm and recommendations for venous thromboembolism prophylaxis in medical patients. Vasc Health Risk Manag. 2007;3(4):533-53. PMid:17969384.

10. Royal College of Obstetrician \& Gynaecologists - RCOG. Reducing the risk of venous thromboembolism during pregnancy and the puerperium. London: Royal College of Obstetricians \& Gynaecologists; 2015. (Green-Top Guideline; no. 37a).

11. Barbar S, Noventa F, Rossetto V, et al. A risk assessment model for the identification of hospitalized medical patients at risk for venous thromboembolism: the Padua Prediction Score.J Thromb Haemost. 2010;8(11):2450-7. http://dx.doi.org/10.1111/j.15387836.2010.04044.x. PMid:20738765.

12. Spyropoulos AC, Anderson FA Jr, Fitzgerald G, et al. Predictive and associative models to identify hospitalized medical patients at risk for VTE. Chest. 2011;140(3):706-14. http://dx.doi.org/10.1378/ chest.10-1944. PMid:21436241.

13. Chopard P, Spirk D, Bounameaux H. Identifying acutely ill medical patients requiring thromboprophylaxis. J Thromb Haemost. 2006;4(4):915-6. http://dx.doi.org/10.1111/j.1538-7836.2006.01818.x. PMid:16634771.

14. Samama MM, Cohen AT, Darmon JY, et al. A comparison of enoxaparin with placebo for the prevention of venous thromboembolism in acutely ill medical patients. N Engl J Med. 1999;341(11):793-800. http://dx.doi.org/10.1056/NEJM199909093411103. PMid:10477777.

15. Caprini JA. Thrombosis risk assessment as a guide to quality patient care. Dis Mon. 2005;51(2-3):70-8. http://dx.doi.org/10.1016/j. disamonth.2005.02.003. PMid:15900257.

16. Rogers SO Jr, Kilaru RK, Hosokawa P, Henderson WG, Zinner MJ, Khuri SF. Multivariable predictors of postoperative venous thromboembolic events after general and vascular surgery: results from the patient safety in surgery study. J Am Coll Surg. 2007;204(6):1211-21. http:// dx.doi.org/10.1016/j.jamcollsurg.2007.02.072. PMid:17544079.

17. Leizorovicz A, Cohen AT, Turpie AG, Olsson CG, Vaitkus PT, Goldhaber SZ. Randomized, placebo-controlled trial of dalteparin for the prevention of venous thromboembolism in acutely ill medical patients. Circulation. 2004;110(7):874-9. http://dx.doi. org/10.1161/01.CIR.0000138928.83266.24. PMid:15289368.

18. Cohen AT, Davidson BL, Gallus AS, et al. Efficacy and safety of fondaparinux for the prevention of venous thromboembolism in older acute medical patients: randomised placebo-controlled trial. BMJ. 2006;332(7537):325-9. http://dx.doi.org/10.1136/ bmj.38733.466748.7C. PMid:16439370.

19. Stuck AK, Spirk D, Schaudt J, Kucher N. Risk assessment models for venous thromboembolism in acutely ill medical patients: a systematic review. Thromb Haemost. 2017;117(4):801-8. http:// dx.doi.org/10.1160/TH16-08-0631. PMid:28150851.

20. Key NS, Khorana AA, Kuderer NM, et al. Venous thromboembolism prophylaxis and treatment in patients with Cancer: ASCO clinical practice guideline update.J Clin Oncol. 2020;38(5):496-520. http:// dx.doi.org/10.1200/JCO.19.01461. PMid:31381464.

21. National Institute for Health and Care Excellence - NICE. Venous thromboembolism in over 16s: reducing the risk of hospital-acquired deep vein thrombosis or pulmonary embolism: NICE guideline [NG89]. London: NICE; 2019. [citado 2020 mar 30]. https://www. nice.org.uk/guidance/ng89/resources

22. Hijazi Z, Oldgren J, Lindbäck J, et al. The novel biomarker-based $\mathrm{ABC}$ (age, biomarkers, clinical history) - bleeding risk score for patients with atrial fibrillation: a derivation and validation study. Lancet. 2016;387(10035):2302-11. http://dx.doi.org/10.1016/ S0140-6736(16)00741-8. PMid:27056738.
23. O'Brien EC, Simon DJN, Thomas LE, et al. The ORBIT bleeding score: a simple bedside score to assess bleeding risk in atrial fibrillation. Eur Heart J. 2015;36(46):3258-64. http://dx.doi.org/10.1093/ eurheartj/ehv476. PMid:26424865.

24. Fang MC, Go AS, Chang Y, et al. A new risk scheme to predict warfarin-associated hemorrhage: the ATRIA (Anticoagulation and Risk Factors in Atrial Fibrillation) study. J Am Coll Cardiol. 2011;58(4):395-401. http://dx.doi.org/10.1016/j.jacc.2011.03.031. PMid:21757117.

25. Pisters R, Lane DA, Nieuwlaat R, de Vos CB, Crijns HJ, Lip GY. A novel user-friendly score (HAS-BLED) to assess 1-year risk of major bleeding in patients with atrial fibrillation: the Euro Heart Survey. Chest. 2010;138(5):1093-100. http://dx.doi.org/10.1378/ chest.10-0134. PMid:20299623.

26. Gage BF, Yan Y, Milligan PE, et al. Clinical classification schemes for predicting hemorrhage: results from the National Registry of Atrial Fibrillation (NRAF). Am Heart J. 2006;151(3):713-9. http:// dx.doi.org/10.1016/j.ahj.2005.04.017. PMid:16504638.

27. Shireman TI, Mahnken JD, Howard PA, Kresowik TF, Hou Q, Ellerbeck EF. Development of a contemporary bleeding risk model for elderly warfarin recipients. Chest. 2006;130(5):1390-6. http:// dx.doi.org/10.1378/chest.130.5.1390. PMid:17099015.

28. Klok FA, Hösel V, Clemens A, et al. Prediction of bleeding events in patients with venous thromboembolism on stable anticoagulation treatment. Eur Respir J. 2016;48(5):1369-76. http://dx.doi. org/10.1183/13993003.00280-2016. PMid:27471209.

29. Ruíz-Giménez N, Suarez C, Gonzalez R, et al. Predictive variables for major bleeding events in patients presenting with documented acute venous thromboembolism: findings from the RIETE Registry. Thromb Haemost. 2008;100(1):26-31. http://dx.doi.org/10.1160/ TH08-03-0193. PMid:18612534.

30. Kuijer PM, Hutten BA, Prins MH, Büller HR. Prediction of the risk of bleeding during anticoagulant treatment for venous thromboembolism. Arch Intern Med. 1999;159(5):457-60. http:// dx.doi.org/10.1001/archinte.159.5.457. PMid:10074953.

31. Beyth RJ, Quinn LM, Landefeld CS. Prospective evaluation of an index for predicting the risk of major bleeding in outpatients treated with warfarin. Am J Med. 1998;105(2):91-9. http://dx.doi. org/10.1016/S0002-9343(98)00198-3. PMid:9727814.

32. Zulkifly $\mathrm{H}$, Lip GYH, Lane DA. Bleeding risk scores in atrial fibrillation and venous thromboembolism. Am J Cardiol. 2017;120(7):1139-45. http://dx.doi.org/10.1016/j.amjcard.2017.06.058. PMid:28800833.

33. Schulman S, Kearon C. Definition of major bleeding in clinical investigations of antihemostatic medicinal products in nonsurgical patients. J Thromb Haemost. 2005;3(4):692-4. http:// dx.doi.org/10.1111/j.1538-7836.2005.01204.x. PMid:15842354.

34. University of Massachusetts Medical School - UMMS [site na Internet]. Worcester, MA: UMASS; 2020. [citado 2020 jun 17]. http:// www.outcomesumassmed.org/IMPROVE/bleeding_risk_score.cfm

35. Hostler DC, Marx ES, Moores LK, et al. Validation of the international medical prevention registry on venous thromboembolism bleeding risk score. Chest. 2016;149(2):372-9. http://dx.doi.org/10.1378/ chest.14-2842. PMid:26867833.

36. Rosenberg DJ, Press A, Rosenberg DJ, et al. External validation of the IMPROVE Bleeding Risk Assessment Model in medical patients. Thromb Haemost. 2016;116(3):530-6. http://dx.doi.org/10.1160/ TH16-01-0003. PMid:27307054.

37. Leonardi MJ, McGory ML, Ko CY. The rate of bleeding complications after pharmacologic deep venous thrombosis prophylaxis: a systematic review of 33 randomized controlled trials. Arch Surg. 2006;141(8):790-7, discussion 797-9. http://dx.doi.org/10.1001/ archsurg.141.8.790. PMid:16924087. 
38. Falck-Ytter Y, Francis CW, Johanson NA, et al. Prevention of VTE in orthopedic surgery patients: antithrombotic therapy and prevention of thrombosis, 9th ed: American College of Chest Physicians Evidence-Based Clinical Practice Guidelines. Chest. 2012;141(2, Supl):e278S-325S. http://dx.doi.org/10.1378/ chest.11-2404. PMid:22315265.

39. Spyropoulos AC, Douketis JD. How i treat anticoagulated patients undergoing an elective procedure or surgery. Blood. 2012;120(15):295462. http://dx.doi.org/10.1182/blood-2012-06-415943. PMid:22932800.

40. Dahl OE, Quinlan DJ, Bergqvist D, Eikelboom JW. A critical appraisal of bleeding events reported in venous thromboembolism prevention trials of patients undergoing hip and knee arthroplasty. J Thromb Haemost. 2010;8(9):1966-75. http://dx.doi.org/10.1111/j.15387836.2010.03965.x. PMid:20586919.

41. Tufano A, Guida A, Di Minno MN, Prisco D, Cerbone AM, Di Minno G. Prevention of venous thromboembolism in medical patients with thrombocytopenia or with platelet dysfunction: a review of the literature. Semin Thromb Hemost. 2011;37(3):267-74. http:// dx.doi.org/10.1055/s-0031-1273090. PMid:21455860.

42. Lim W. Prevention of thrombosis in antiphospholipid syndrome. Hematology. 2016;2016(1):707-13. http://dx.doi.org/10.1182/ asheducation-2016.1.707. PMid:27913550.

43. Farge D, Bounameaux $H$, Brenner B, et al. International clinical practice guidelines including guidance for direct oral anticoagulants in the treatment and prophylaxis of venous thromboembolism in patients with cancer. Lancet Oncol. 2016;17(10):e452-66. http:// dx.doi.org/10.1016/S1470-2045(16)30369-2. PMid:27733271.

44. Key NS, Bohlke K, Falanga A. Venous thromboembolism prophylaxis and treatment in patients with cancer: ASCO clinical practice guideline update summary. J Oncol Pract. 2019;15(12):661-4. http://dx.doi.org/10.1200/JOP.19.00368.
45. Aursulesei V, Costache II. Anticoagulation in chronic kidney disease: from guidelines to clinical practice. Clin Cardiol. 2019;42(8):774-82. http://dx.doi.org/10.1002/clc.23196. PMid:31102275.

46. Singh S, Haut ER, Brotman DJ, et al. Pharmacologic and mechanical prophylaxis of venous thromboembolism among special populations. Rockville: Agency for Healthcare Research and Quality; 2013.

Correspondência Marcos Arêas Marques Universidade do Estado do Rio de Janeiro (UERJ) Rua Assunção 217/704 - Botafogo CEP 22251-030 - Rio de Janeiro (RJ), Brasil Tel.: (21) 99859-0160 E-mail:mareasmarques@gmail.com

Informaç̧ões sobre os autores MCC - Professora, Departamento de Clínica Médica, Universidade Federal do Rio de Janeiro (UFRJ). MAM - Angiologista, Unidade Docente Assistencial de Angiologia, Hospital Universitário Pedro Ernesto, Universidade do Estado do Rio de Janeiro (UERJ).

Contribuições dos autores Concepção e desenho do estudo: MCC Análise e interpretação dos dados: MCC, MAM Coleta de dados: MCC Redação do artigo: MCC, MAM Revisão crítica do texto: MCC, MAM Aprovação final do artigo*: MCC, MAM Análise estatística: MCC Responsabilidade geral pelo estudo: MCC

*Todos os autores leram e aprovaram a versão final submetida ao J Vasc Bras. 